\title{
Post Eradication Assessment of Hepatic Fibrosis in Hepatitis C
}

\author{
Hanan Hamed Soliman \\ Tropical Medicine Department, Faculty of Medicine, Tanta University, Egypt
}

Mobile:

$+20100469443$

E mail:

Hanan.soliman@med.t anta.edu.eg

hanansol@gmail.com

Key words:

Liver biopsy, ABRI,

FIB4,Fibroscan,

VCTE, LSM, DAAS hepatitis $C$
Histological analysis of liver tissue gives us insight about a wide varity of liver diseases starting with acute and chronic hepatitis, alcoholic and nonalcoholic steatosis, metabolic and storage diseases and hepatic neoplasm. Liver biopsy has 2 main indications; either to diagnose the disease or to stage its severity [1].

In chronic hepatitis $\mathrm{C}(\mathrm{CHC})$ infected patient, biopsy is used mainly for staging. Chronic HCV infection can induce significant fibrosis, cirrhosis, and eventually, hepatocellular carcinoma. Knowing the histologic stage and grade of the disease is useful in predicting the short- and long-term prognosis of the patient. One decade ago a baseline liver biopsy prior to treating patients for chronic hepatitis $\mathrm{C}$ with interferon therapy was mandatory to stage the fibrosis and justify treatment. Although liver biopsy is the gold standard to stage the fibrosis and necroinflammatory changes in chronic $\mathrm{HCV}$, but it is an invasive procedure, costly, carry risk of morbidity, and liable to sampling error due to heterogeneity in fibrosis distribution. The misclassification of biopsy due to observer agreement variability especially between F2 and F1 was reported in more than $25 \%$ of cases [2-4].

Moreover, hepatic fibrosis was formerly thought to be irreversible. Now, it is recognized as a dynamic process with the potential for significant resolution. As liver biopsy provides only a snapshot of this dynamic process, which necessitates repeating the biopsy for follow up, the need for noninvasive means to measure changes in fibrosis was initiated. Increasing attention is paid to non-invasive tests for detecting fibrosis in those patients especially in last decade. This advent of noninvasive approaches for assessment of liver fibrosis, combined with evolution of oral direct-acting antiviral (DAAs) drugs. DAAs with their high efficacy and much less side effects has eliminated the need for liver biopsy to differentiate mild from significant $(\geq \mathrm{F} 2)$ disease prior to antiviral therapy for CHC. Anyhow, we still need to trace the dynamic change in pathology after viral eradication. Here, the non-invasive modalities are of great help in follow up of responders to detect the change in the pathology [5-6].

The non-invasive approaches for assessment of liver fibrosis include two main categories: (1) imaging modalities able to measure liver stiffness; either shared wave elastography, transient elastography (fibroscan) or MRI elastography [7]; (2) biochemical serum markers which may be direct or indirect markers. Direct markers are fragments of the liver matrix produced by hepatic stellate cells during the process of extracellular matrix remodeling. Indirect markers are more variable and include; molecules released due to liver inflammation such as ALT, AST, GGT; molecules synthesized, excreted or regulated by the liver such as albumin, bilirubin, INR; and markers of processes commonly associate liver function impairment, such as platelet count. Several indices and scores were developed by combining some direct or indirect markers with routine investigations in 
equations. Most popular are fibrotest or fibrosure, hepascore, APRI, and FIB4 [8-11].

Fibroscan (TE) was found to be more accurate compared to share wave elastography in detecting liver fibrosis in relation to pathology [12]. In an Egyptian study, El Sadaany et al.,2016 [13] found Fibroscan to correlate with histopathology in moderate (F2-F3), but not mild (F1) fibrosis in $\mathrm{CHC}$ patients. They also reported the LSM degree of fibrosis to regresses significantly in $\mathrm{HCV}$ responders on anti-viral INF based therapy and recommend the use of fibroscan for follow up of these patients [13].

Fibroscan examination for detection of fibrosis as surrogate for liver biopsy is accepted and recommended by many guidelines $[\mathbf{1 4 , 6 ]}$.

Eissa et al., in a study published in the current issue of the in Afro-Egypt $\mathrm{J}$ Infect Endem Dis entitled "Liver Fibrosis Assessment in Cases of Chronic Hepatitis C after Direct Acting Antivirals Therapy using Aspartate Aminotransferase to Platelet Ratio Index and Transient Elastography" evaluated changes in liver fibrosis among chronic $\mathrm{HCV}$ patients after DAAs therapy using fibroscan and APRI. They compared the scores of each patient before and one year after a full course of DAAs therapy. They found that liver stiffness measurement was markedly decreased from 10.6 to $6 \mathrm{kPa}$ after 12 months of completion of DAAs therapy, with significant reduction of stage of fibrosis in more than $76 \%$ of their patients. The reduction of fibrosis stages had occurred in $87.50 \%$ of patients with F2 stage, $87.5 \%$ of patients with F3 stage, and $76.5 \%$ of patients with $\mathrm{F} 4$ stage. A relevant study on 300 Egyptian HCV patients showed reduction in fibrosis in; $46.7 \%$ and $49.3 \%$ of patients with moderate fibrosis, and $89 \%$ and $78.7 \%$ of patients with advanced fibrosis after one year of interferon containing and interferon free DAAs regimens respectively [15] which emphasize a significant reduction of LSM after DAAs therapy.

Interestingly, Eissa et al., reported that all of their patients with liver fibrosis stages (F2 or F3) did not show the progression of their liver fibrosis stages after full course of DAAs therapy. This finding suggest DAAs therapy to prevent progression of fibrosis; most probably because eradication of HCV prevent further damage and subsequent fibrosis.
Authors also reported that the median APRI value was significantly reduced from 1.12 at the baseline to 0.42 (range, 0.12-2.00) after 12 months of DAAs therapy. APRI is calculated as (AST/upper limit of normal range)/platelet count $(109 / \mathrm{L}) \times 100$. A large meta-analysis suggested APRI to have moderate degree of accuracy to identify hepatitis C-related fibrosis [16].

The combination of LSM and simple fibrosis scores, like APRI in this study, has the advantage of improving the moderate accuracy of these tests. It can reduce the proportion of patients with indeterminate results.

To conclude, although liver biopsy is still the gold standard in staging fibrosis in $\mathrm{HCV}$, it is not widely accepted now.Non-invasive tests to detect fibrosis can be adopted in $\mathrm{CHC}$ to evaluate and follow the fibrosis progression after therapy. Combining two or more non-invasive tests to evaluate fibrosis is more reliable. The results of the current study support the idea of liver fibrosis reversibility after treatment of the underlying etiology of chronic liver disease especially in patients with $\mathrm{CHC}$.

\section{REFERENCES}

1. Boyd A, Cain O, Chauhan A, Webb GJ. Medical liver biopsy: background, indications, procedure and histopathology. Frontline Gastroenterol. 2020 Jan; 11(1):40-47.

2. Bedossa P, Dargere D, Paradis V. Sampling variability of liver fibrosis in chronic hepatitis C. Hepatology 2003; 38 (2003): 1449-1457.

3. Rockey D, Caldwell S, Goodman Z, Nelson R, Smith A. Liver biopsy Hepatology, 49 (2009), pp. 1017-1044

4. Rousselet MC, Michalak S, Dupré F, Croué A, Bedossa P, Saint-André JP, Calès P; Hepatitis Network 49. Sources of variability in histological scoring of chronic viral hepatitis. Hepatology. 2005 Feb; 41(2):257-64.

5. Wei L, Lok AS. Impact of new hepatitis C treatments in different regions of the world. Gastroenterology 2014; 146:1145-50.

6. European Association for Study of Liver, Asociacion Latinoamericana para el Estudio del Higado. EASL-ALEH clinical practice guidelines: non-invasive tests for evaluation of liver disease severity and prognosis. J Hepatol 2015; 63: 23764. 
7. Boursier J, Zarski JP, de Ledinghen V, Rousselet MC, Sturm N, Lebail B et al. Determination of reliability criteria for liver stiffness evaluation by transient elastography. Hepatology 2013; 57: 1182-1191.

8. Grigorescu M. Noninvasive biochemical markers of liver fibrosis. Journal of gastrointestinal and liver diseases 2006; 15(2):149-59

9. Lin W, Wu G, Li S, Weinberg EM, Kumthip K, Peng LF, et al . Performance of the aspartate aminotransferase-to-platelet ratio index for the staging of hepatitis C-related fibrosis: an updated metaanalysis. Hepatology 2010; 53: 726-736.

10. Sterling RK, Lissen E, Clumeck N, Sola R, Correa MC, Montaner J,et al. APRICOT Clinical Investigators:Development of a simple noninvasive index to predict significant fibrosis in patients with HIV/HCV coinfection. Hepatology 2006; 43: 1317-25.

11. Galal G, Abd-El-Fatah M, Kamel S. Non-invasive methods for diagnosis of hepatic fibrosis in chronic hepatitis B patients. Sohag Medical Journal 2018; 22(3), 245-251.

12. Cassinotto C, Lapuyade B, Mouries A, Hiriart JB, Vergniol J, Gaye D, et al. Non-invasive assessment of liver fibrosis with impulse elastography: comparison of Supersonic Shear Imaging with ARFI and FibroScan®. J Hepatol 2014 Sep; 61(3):550-7.

13. El Saadany S, Soliman H, Ziada D, Hamisa M, Hefeda M ,Selim A Goraba H. Fibroscan versus liver biopsy in the evaluation of response among the Egyptian HCV infected patients to treatment The Egyptian Journal of Radiology and Nuclear Medicine 2016; 47 (Issue 1): 1-7.

14. AASLD/IDSA HCV Guidance Panel. Hepatitis C guidance: AASLDIDSA recommendations for testing, managing, and treating adults infected with hepatitis C virus. Hepatology 2015; 62(3):932-954.

15. Soliman H, Ziada D, Salama M, Hamisa M, Badawi R, Hawash N, et al. Predictors for Fibrosis Regression in Chronic HCV Patients after the Treatment with DAAS: Results of a Real-world Cohort Study. Endocr Metab Immune Disord Drug Targets 2020; 20(1):104-111.

16. Lin W, Wu G, Li S, Weinberg EM, Kumthip K, Peng LF,et al. Performance of the aspartate aminotransferase-to-platelet ratio index for the staging of hepatitis C-related fibrosis: an updated metaanalysis. Hepatology 2010; 53:726-36. 